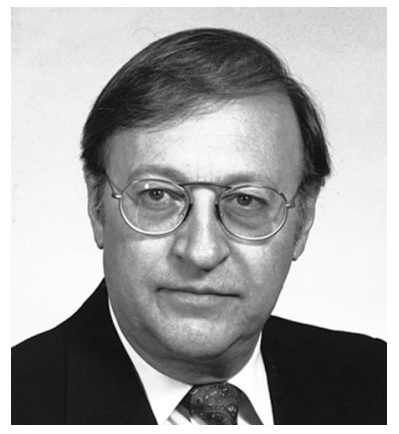

\section{Is referencing an art or an obligation?}

Doing research is a big deal. Finding new explanations for phenomena is a big deal. Finding new ways to do things is also a big deal. Worldwide, research scientists work hard to give a better understanding to natural phenomena, and to develop new theories that will give a more complete picture of experimental results. Applied scientists enthusiastically try to find applications or new ways of using well-known knowledge.

In this quest, worldwide knowledge is based upon published works that have accumulated over time. Very often these publications can be difficult to find, since researchers work in many different languages and all have the right and obligation to publish in their own language.

This problem has existed since the beginning of the era of publication. However, this problem may have increased with the development of research capabilities in all countries. Many researchers were isolated, with poor access to the pool of existing and/or published knowledge. This is why it is quite common to find the same piece of work done or published in different languages, each stating that they are the first to do this work.

However, I remember my thesis supervisor advising us to go to the library to search and read related work in such a way that we would be able to give credit where it is due. I remember his vast knowledge of scientific literature and his references to many phenomena or solutions by the names of their discoverers and inventors. I understand that this might be not possible today, with the explosion of published papers and the increased number of journals.

Nowadays, we all have access to databases full of information, which allow everyone to get a complete view of the literature related to a particular problem. This great accessibility to other work allows a more rapid development of knowledge and a rapid growth of new applications. I think that all of us can see this effect.

However, this greater accessibility is not always properly used. In fact, often researchers, either intentionally or by lack of reviewing previous work, do not cite prior work. Often, the work is cited using detour, and readers can find, hidden inside another reference, the original work that led to the published research. There are also those who cite, but group many references in a bunch under a single heading, thereby effectively hiding the original work.

There is also another phenomenon in which authors want to please some potential reviewers by deliberately citing their work as opposed to the field's original work. It is not my intent to say that authors must not cite these particular works, but by bypassing the original, they tend to give inflated credit to work that is not the true original.

This editorial aims to point out that researchers have wonderful instruments available to them, which allow them to do extensive reviews of literature. However, it seems that many do not use these instruments to complete their knowledge and avoid duplication of previously published work. Researchers are pressed to get new-or what may be seen as new-results to please their supervisors, their granting bodies, etc. I personally hope that we can all make better use of the available literature search capabilities that technology has given us. It is very frustrating to read articles where a part of the reported work-or even all the work - has already been published.

The problem with references in papers submitted for publication isn't worse than it was in the past, but surprisingly, it is not less than it was before the development of high-tech search capabilities. It is a pity.

Hopefully this editorial will put forth the question of whether referencing is an art or an obligation. Maybe we will discover that it is an obligation. 